1 Universidade Federal da Bahia (UFBA), Instituto Multidisciplinar em Saúde - Vitória da Conquista (BA), Brasil.

maiaufba@ufba.br

2 Fundação Oswaldo Cruz (Fiocruz), Escola Nacional de Saúde Pública Sergio Arouca (Ensp) - Rio de Janeiro (RJ), Brasil. giovanel@ensp.fiocruz.br

\section{Estratégia Saúde da Família na coordenação do cuidado em região de saúde na Bahia}

\author{
Family Health Strategy in care coordination in a health \\ administrative region in Bahia, Brazil
}

Adriano Maia dos Santos'1, Ligia Giovanella²
RESUMO Este artigo analisa limites e possibilidades de coordenação do cuidado por Equipes de Saúde da Família em região de saúde, Bahia. Trata-se de estudo de caso com abordagem qualitativa, numa região de saúde que abrange dezenove municípios. Resultados emergiram das análises de dados coletados em 17 entrevistas semiestruturadas com gestores, nove grupos focais, observações e documentos. Na região, equipes têm dificuldades na retaguarda assistencial, limitando a continuidade do cuidado e a resolubilidade dos casos que precisam de suporte especializado. Os fluxos comunicacionais incipientes entre diferentes pontos da rede não viabilizam a coordenação do cuidado por Equipes de Saúde da Família.

PALAVRAS-CHAVE Atenção Primária à Saúde; Controle de acesso; Regionalização; Continuidade da assistência ao paciente.

ABSTRACT This qualitative case study examines the limitations and scope of care coordination by Family Health Teams in a health administrative region comprising 19 municipalities located in the State of Bahia, Brazil. The results emerged from analyses and correlation of data collected from 17 semi-structured interviews of policy makers and managers, nine focal groups, observations and documents. The region's Family Health Teams face difficulties in providing care support, which limits care continuity and resolution rates in cases needing specialised support. Incipient communication flows among the network points do not enable coordinating care by Family Health Teams.

KEYWORDS Primary Health Care; Gatekeeping; Regional health planning; Continuity of patient care. 


\section{Introdução}

Dentre os argumentos em defesa da integração dos serviços e coordenação do cuidado para organização dos sistemas de saúde, destacam-se a necessidade de reduzir a segmentação e fragmentação da atenção, garantir a equidade no acesso, aumentar a eficiência e a qualidade dos serviços, evitar a sobreposição desnecessária, onerosa e iatrogênica em intervenções médicas, direcionar os serviços e distribuir os profissionais adequadamente entre diferentes níveis (SALTMAN; RICO; BOERMA, 2006). Para tanto, deve-se buscar a gestão da clínica e a continuidade do cuidado das condições crônicas, reduzindo a hospitalização desnecessária por meio de uma atenção balanceada, com adequação da oferta e organização dos fluxos entre serviços especializados e a atenção de primeiro nível (GREB ET AL., 2009), controlar os custos, além de favorecer fluxo comunicacional, atenção ao longo do tempo e continuidade do cuidado (HAGGERTY ET AL., 2003).

O envelhecimento populacional, a crescente necessidade de enfrentamento das condições crônicas, as comorbidades, as novas modalidades de diagnóstico e terapia, a crescente especialização e subespecialização dos profissionais, a demanda por cuidados continuados e os custos na área da saúde têm exposto a ineficiência dos sistemas fragmentados e descoordenados para lidarem com tais questões (MUR-VEEMAN; VAN RAAK; PAULUS, 2008).

A coordenação do cuidado, na Europa, por exemplo, tem logrado maior sucesso entre países que estabeleceram a Atenção Primária à Saúde (APS) como porta de entrada e o médico generalista como coordenador do cuidado (gatekeeper) (GIOVANELLA, 2011; SALTMAN; RICO; BOERMA, 2006). Ademais, o sucesso na coordenação via APS depende, também, do prestígio que médicos generalistas desfrutam entre o coletivo de médicos especialistas e na sociedade (GÉRVAS ET AL., 2007). Não obstante, as principais dificuldades para a coordenação de serviços de saúde ocorrem pela assimetria da informação. Além disso, as decisões médicas são de difícil julgamento e os resultados sanitários não são fáceis de medir, pois nem sempre são imediatos e não estão atrelados unicamente aos serviços de saúde (GÉRVAS; RICO, 2005).

A coordenação é um atributo da APS e funciona como um amálgama, pois potencializa e viabiliza os cuidados continuados, articula os diferentes pontos do sistema para a integralidade e desburocratiza a função de primeiro contato, ou seja, parte do reconhecimento que as diferentes necessidades de saúde e as possibilidades de resolução não podem ser alcançadas apenas pela equipe de APS (STARFIELD, 2002).

Estudos defendem a posição da APS como porta de entrada preferencial e coordenadora do cuidado (STARFIELD; SHI; MACINKO, 2005; GÉRVAS; PEREZ FERNANDEZ, 2005). Assim, a atuação dos profissionais, precipuamente médicos, com capacidade para resolver problemas nas unidades de saúde e encaminhar adequadamente os usuários para serviços de referência somente depois de esgotadas as possibilidades terapêuticas por meio do mecanismo gatekeeping (controle de acesso) é um sinalizador de qualidade e adequação da posição da APS na rede de atenção, essencialmente quando essa atuação permite a coordenação do cuidado entre níveis de atenção, busca a resolução do problema dos usuários com continuidade e não se caracteriza como barreira de acesso oportuno (STARFIELD; SHI; MACINKO, 2005; GÉRVAS; FERNÁNDEZ, 2005).

O mecanismo de gatekeeping não é uma resposta inequívoca, visto que a maioria dos estudos disponíveis avaliam tal mecanismo na perspectiva do managed care estadunidense, focando na redução de gasto e diminuição na utilização de serviços especializados, ao passo que investigações que relacionam essa função em sistemas universais e o impacto na saúde e na qualidade da atenção prestada ao usuário são muitas vezes inconclusivas (GARRIDO; ZENTNER; BUSSE, 2011). Em 
países europeus (Bélgica, Espanha e Suíça) e no Canadá, houve melhor uso dos serviços especializados, menor quantidade de intervenções desnecessárias e coordenação adequada para o melhor cuidado do usuário, resultantes da presença do médico generalista na função de filtro (gatekeeper) e APS como porta de entrada preferencial (GÉRVAS; FERNÁNDEZ, 2005).

Em traços gerais, nos países europeus com sistemas universais, organizados por serviços nacionais de saúde, é comum que a porta de entrada seja uma unidade de APS, por meio de um profissional de primeiro contato, frequentemente médico, que utiliza a prerrogativa de gatekeeper no encaminhamento dos usuários aos demais pontos da rede de saúde, facilitando, assim, a coordenação do cuidado por um médico generalista (GÉRVAS; FERNÁNDEZ, 2005; ALMEIDA ET AL., 2013). Por outro lado, mesmo países com forte organização via gatekeeper, com a APS posicionada formalmente como serviço de primeiro contato, há procura e contato inicial via emergência hospitalar (GIOVANELLA, 2006).

$\mathrm{O}$ argumento deste artigo é o de que as atribuições do médico generalista na função de gatekeeper não devem restringir-se ao controle de acesso. No âmbito do Sistema Único de Saúde (SUS), espera-se o fortalecimento do papel do generalista com competência para coordenação clínica do cuidado a um paciente ou grupo de pessoas entre a APS e os demais níveis de atenção por meio de reconhecimento das necessidades de saúde dos usuários, responsabilizando-se pelo encaminhamento interno ou para outro serviço mais adequado, bem como por meio de contato direto com outros profissionais especialistas para complementar as informações e aumentar a resolubilidade, a fim de prosseguir com o acompanhamento continuado. Tal perspectiva requer sistemas orientados pela APS (CONILL; FAUSTO; GIOVANELLA, 2010; KRINGOS ET AL., 2010), serviços integrados em rede (ALMEIDA ET AL., 2013; VÁZQUEZ ET AL., 2009) e compartilhamento de informações clínicas (SHOEN ET AL., 2012) entre profissionais para promover a continuidade do cuidado entre níveis (HERNÁNDEZ, 2010).

Diante dos desafios apresentados no cenário internacional, este artigo analisa os limites e possibilidades de coordenação do cuidado e controle de acesso aos serviços especializados (gatekeeping) por Equipes de Saúde da Família (EqSF) em região de saúde na Bahia, Brasil.

\section{Metodologia}

Trata-se de estudo de caso, com abordagem qualitativa, realizado na região de saúde de Vitória da Conquista, que abrange dezenove municípios da Bahia, entre 2011 e 2012. O Decreto 7.508 considera as regiões de saúde como territórios privilegiados para integração dos serviços de saúde e coordenação do cuidado (BRASIL, 2011). Na Bahia, existem 28 regiões de saúde e, para cada uma, por meio a Resolução n. ${ }^{\circ} 275$ (BAHIA, 2012), foi instituída uma Comissão Intergestores Regional (CIR). As CIR são instâncias de articulação entre gestores estaduais e municipais que devem pactuar, de maneira coordenada e solidária, a distribuição dos serviços e profissionais, visando garantir amplo acesso à população a diferentes níveis de atenção à saúde na região de sua abrangência.

Na pesquisa, o conceito central é a coordenação do cuidado, compreendida como a articulação entre diversos serviços, ações e profissionais relacionados à atenção em saúde, de forma que, independentemente do local onde seja prestado, esteja sincronizado e voltado ao alcance de um objetivo comum (HOFMARCher; OXLEY; RUSTICELLI, 2007). Para viabilizar a coordenação, o mecanismo gatekeeping é estratégico e, nesta perspectiva, distinguimos três tipologias nos serviços de APS: tipo 1 - gatekeeping como regulador burocrático; tipo 2 - gatekeeping sem regulação assistencial; e tipo 3 - gatekeeping como coordenador do cuidado. O primeiro tipo, por ser burocrático, não distingue a essência dos serviços, 
apenas tenta garantir racionalidade dos encaminhamentos clínicos, tendo, no fundo, uma visão mais gerencial e econômica do que uma centralização no cuidado, ainda que, no final, o cuidado possa ser o resultado. $\mathrm{O}$ segundo tipo não segue diretrizes clínicas, desempenha a função de filtro mediado por interesse ou conveniência do profissional, por pressão do usuário ou interferência política, não essencialmente, conforme a necessidade clínica do usuário e sem avaliação de cotas (regulação) ou prioridades terapêuticas. Por fim, a terceira opção, teria a incumbência de olhar atentamente cada usuário, avaliar as alternativas terapêuticas mais adequadas e organizar o fluxo entre a EqSF e outros profissionais dos diferentes pontos da rede conforme a necessidade de saúde das pessoas, mantendo-se informado sobre o itinerário e a terapêutica dos usuários sob cuidado externo à Estratégia Saúde da Família (ESF), que, no final, pode gerar, também, economia nos gastos.

As fontes de dados (quadro 1) provieram de 17 entrevistas semiestruturadas com gestores (estadual, regional e municipal), nove grupos focais (quatro grupos com trabalhadores da saúde, quatro grupos com usuários e um grupo com apoiadores institucionais da Secretaria Estadual de Saúde da Bahia Sesab) registros de observações na CIR (sete reuniões em outubro/2011 a março/2012), em Unidades de Saúde da Família (USF), Centrais de Marcação de Consultas (CMC), complementados por documentos: atas da CIR (2011-2012) e Relatórios Anuais de Gestão (RAG) de 2011, dentre outros. Para garantir a privacidade, fragmentos de fala foram codificados como 'G' (gestores), seguido do plano de atuação (estadual 'E', regional 'R' ou municipal 'M'), 'GF-G' (grupo focal com gestores), 'GF-T' (grupo focal com trabalhadores da saúde) e 'GF-U' (grupo focal com usuários).

Os gestores entrevistados ocupavam posição estratégica nas secretarias de saúde municipais e estadual ou na diretoria regional de saúde, sendo escolhidos mediante observação de atuação (ativa e frequente) nas reuniões da CIR e por indicação de informantes-chave que sugeriram os sujeitos que acumulavam experiência consolidada nos respectivos cargos ou com grande representação ou influência entre os pares.

As entrevistas contemplaram gestores de diferentes municípios da região e que conheciam e atuavam na organização regional. As observações da CIR permitiram o acompanhamento da governança regional do conjunto dos municípios e foram registradas em diário de campo com destaque aos aspectos discutidos em plenária, pertinentes à coordenação do cuidado.

Para realização dos grupos focais, foram eleitos quatro municípios para ilustrar a diversidade de estratégias na organização dos fluxos assistenciais, intencionando-se apreender as articulações que possibilitavam ou não a coordenação do cuidado na região de saúde. O critério de seleção dos municípios deu-se pelo cruzamento de informações consideradas relevantes durante a exploração do campo: 1) indicação de informantes-chave: municípios que apresentam a melhor organização de serviços de saúde; 2) observação em reuniões da CIR: sete encontros; 3) participação ativa do gestor municipal na CIR; 4) cobertura da ESF: >70\%; 5) municípios de porte populacional distintos: I - até 10.000 hab.; II - de 10.001 a 25.000 hab.; III - de 25.001 a 50.000hab.; 6) inclusão do município sede da região de saúde: independentemente da cobertura da ESF.

Realizaram-se quatro grupos focais com médicos e enfermeiros da ESF (GF-T), quatro grupos focais com usuários dos serviços de saúde (GF-U), um em cada município selecionado na região e um grupo focal com apoiadores institucionais (gestores) da Sesab.

Para formação dos grupos focais com trabalhadores, elegeram-se as seguintes características: 1) trabalhadores considerados pela gestão local como profissionais que desenvolviam um bom trabalho na ESF; 2) profissionais de diferentes Unidades de Saúde da Família; 3) médicos e enfermeiros; 4) grupo 
composto por, no mínimo, quatro trabalhadores, sendo obrigatória a presença de pelo menos um médico; 5) profissionais com, no mínimo, um ano de experiência na ESF. Para conformação do grupo focal com usuários, foram utilizadas as seguintes características: 1) usuários que frequentavam rotineiramente a USF; 2) usuários que necessitaram de consultas ou procedimentos especializados em Vitória da Conquista, município de referência da pesquisa; 3) grupo composto por, no mínimo, quatro usuários. Formou-se um grupo focal de usuários por município, sendo que, para cada município, o grupo de usuário foi selecionado em apenas uma USF. Para facilitar o contato, a USF foi selecionada por sorteio dentre os participantes do grupo focal de profissionais.

A intenção não foi o julgamento de cada município isoladamente, mas a compreensão de processos que acontecem na rede regional, por meio de cenários representativos, analisando-se as dimensões político-institucional, organizacional e das práticas.

Ressalta-se que a coleta de dados foi extensa, mas não foi possível trazer tudo pormenorizado no recorte deste artigo. Desta forma, este artigo traz, nos resultados, alguns fragmentos mais representativos de entrevistas e grupos focais, bem como, sínteses narrativas dos demais depoimentos, documentos e observações.

\begin{tabular}{|c|c|c|c|c|c|c|c|}
\hline $\begin{array}{c}\text { Técnicas de } \\
\text { coleta de } \\
\text { dados }\end{array}$ & Sujeitos & Cenários & $\begin{array}{c}\text { Tempo ou } \\
\text { período de } \\
\text { coleta }\end{array}$ & CIR & Municípios & Estado & Região \\
\hline Entrevistas & Gestor & $\begin{array}{l}\text { Municipal } \\
\text { Regional } \\
\text { Estadual }\end{array}$ & $21 \mathrm{~h} 44 \mathrm{~min}$ & - & 11 sujeitos & 3 sujeitos & 3 sujeitos \\
\hline \multirow{3}{*}{ Grupo Focal } & Gestor ${ }^{\star}$ & Estadual & 1h33min & - & - & 4 sujeitos & - \\
\hline & $\begin{array}{l}\text { Trabalha- } \\
\text { dor }\end{array}$ & Municipal & $6 \mathrm{~h} 45 \mathrm{~min}$ & - & $\begin{array}{l}15 \text { sujeitos da } \\
\text { ESF, sendo } \\
6 \text { médicos e } \\
9 \text { enfermeiros }\end{array}$ & - & - \\
\hline & Usuário & Municipal & $4 \mathrm{~h} 27 \mathrm{~min}$ & - & $\begin{array}{l}21 \text { sujeitos } \\
\text { da ESF }\end{array}$ & - & - \\
\hline Observação & - & $\begin{array}{l}\text { Municipal } \\
\text { Regional } \\
\text { Estadual }\end{array}$ & $\begin{array}{l}\text { setembro } \\
\text { a marcco de } \\
2011\end{array}$ & $\begin{array}{l}19 \text { su- } \\
\text { jeitos e } \\
7 \text { ob- } \\
\text { serva- } \\
\text { ções }\end{array}$ & $\begin{array}{l}7 \text { Unidades } \\
\text { de Saúde } \\
\text { da Família, } \\
4 \text { Centrais de } \\
\text { Marcação de } \\
\text { Consultas e } \\
4 \text { Centros de } \\
\text { Especialidades }\end{array}$ & - & - \\
\hline Documentos & - & $\begin{array}{l}\text { Municipal } \\
\text { Regional } \\
\text { Estadual }\end{array}$ & $\begin{array}{l}2009 \text { a } \\
2012\end{array}$ & $\begin{array}{l}\text { Regi- } \\
\text { mento } \\
\text { Interno } \\
(2012) \\
\text { Atas } \\
(2012)\end{array}$ & $\begin{array}{l}\text { Plano Munici- } \\
\text { pal de Saúde } \\
\text { (2011) e } \\
\text { Relatório } \\
\text { Anual de Ges- } \\
\text { tão (2011) }\end{array}$ & $\begin{array}{l}\text { Agenda Estratégica } \\
\text { da Saúde (2009- } \\
\text { 2010), } \\
\text { Plano Diretor de } \\
\text { Regionalização } \\
\text { (2010), } \\
\text { Plano Estadual de } \\
\text { Saúde (2012-2015), } \\
\text { Política Estadual } \\
\text { de Atenção Básica } \\
\text { (2011), } \\
\text { Relatório Anual de } \\
\text { Gestão (2010 e } \\
\text { 2011) }\end{array}$ & $\begin{array}{l}\text { Relatório } \\
\text { Anual da } \\
\text { Atenção } \\
\text { Básica } \\
\text { (2011) }\end{array}$ \\
\hline
\end{tabular}


A opção para interpretar o material coletado foi a análise de conteúdo temática (MINAYO, 2004). Fez-se entrecruzamento entre diferentes informações coletadas, complementado-as com os documentos, identificando-se convergências e divergências para produção de análise compreensiva e crítica. A análise teve por objetivo interpretar os sentidos elaborados pelos discursos dos sujeitos da pesquisa, atentando-se às contradições que lhes são inerentes. Para tanto, utilizou-se uma matriz com duas dimensões para análise da coordenação do cuidado: 1) organização da Estratégia Saúde da Família; e 2) mecanismos de coordenação do cuidado (quadro 2).

Quadro 2. Matriz para análise da coordenação do cuidado pela Estratégia Saúde da Família, região de saúde de Vitória da Conquista, Bahia, 2012

\begin{tabular}{|c|c|c|}
\hline Dimensões de análise & Componentes & Indicadores \\
\hline \multirow{2}{*}{$\begin{array}{l}\text { Organização da Estratégia Saúde } \\
\text { da Família (ESF) }\end{array}$} & $\begin{array}{l}\text { Porta de entrada preferencial } \\
\text { na rede }\end{array}$ & $\begin{array}{l}\text { Cobertura da ESF nos municípios da região de } \\
\text { saúde } \\
\text { Posição da ESF na rede regional } \\
\text { Mecanismos de acesso à Unidade de Saúde da } \\
\text { Família (USF) } \\
\text { USF como serviço de busca regular e primeiro } \\
\text { contato }\end{array}$ \\
\hline & Mecanismos de acesso à ESF & $\begin{array}{l}\text { Exigência e cumprimento da carga horária } \\
\text { por médicos das Equipes de Saúde da Família } \\
\text { (EqSF) } \\
\text { Existência de equipe mínima completa na USF } \\
\text { Número de pessoas cadastradas por EqSF } \\
\text { Atendimento à demanda espontânea }\end{array}$ \\
\hline \multirow{3}{*}{$\begin{array}{l}\text { Mecanismos de coordenação do } \\
\text { cuidado }\end{array}$} & $\begin{array}{l}\text { Oferta de serviços e meca- } \\
\text { nismo de acesso à rede espe- } \\
\text { cializada }\end{array}$ & $\begin{array}{l}\text { Disponibilidade na oferta de vagas no centro } \\
\text { público de especialidades } \\
\text { Mecanismo usado pela EqSF para marcação de } \\
\text { consultas e exames especializados } \\
\text { Existência e funcionamento de central informa- } \\
\text { tizada de regulação e marcação de consultas e } \\
\text { exames especializados } \\
\text { Interferências políticas no fluxo assistencial e } \\
\text { na gestão clínica } \\
\text { Monitoramento de lista de espera para consul- } \\
\text { ta e exames especializados pela EqSF }\end{array}$ \\
\hline & $\begin{array}{l}\text { Instrumentos de comunicação } \\
\text { entre níveis de atenção }\end{array}$ & $\begin{array}{l}\text { Tipo de prontuário para registro de consulta } \\
\text { clínica } \\
\text { Disponibilidade de informações clínicas entre } \\
\text { profissionais da rede } \\
\text { Existência e uso de instrumentos de referência } \\
\text { e contrarreferência entre EqSF e serviços es- } \\
\text { pecializados } \\
\text { Formas de contato formal e informal entre } \\
\text { EqSF e profissionais de diferentes pontos da } \\
\text { rede } \\
\text { Articulação entre médicos especialistas e EqSF }\end{array}$ \\
\hline & Função de filtro (gatekeeper) & $\begin{array}{l}\text { Clínica desenvolvida pela EqSF (centrada no } \\
\text { usuário, na doença ou procedimento) } \\
\text { Formação adequada dos profissionais para } \\
\text { trabalho na EqSF } \\
\text { Resolução do problema de saúde em atendi- } \\
\text { mento prestado na ESF } \\
\text { Ações realizadas quando não há resolução do } \\
\text { problema de saúde na ESF } \\
\text { Articulação entre médicos especialistas e EqSF } \\
\text { Função do médico no controle de acesso às } \\
\text { especialidades (gatekeeper) } \\
\text { Profissionais dos demais níveis da rede reco- } \\
\text { nhecem/respeitam o papel de filtro do médico }\end{array}$ \\
\hline
\end{tabular}

Fonte: Elaboração própria. 
A pesquisa foi aprovada pelo Comitê de Ética em Pesquisa da Ensp (Parecer n. ${ }^{\circ}$ 207/11).

\section{Resultados}

\section{1) Organização da Estratégia Saúde da Família}

Na região de saúde, o fluxo para que usuários consigam realizar consultas, exames e procedimentos em serviços especializados dá-se, sobretudo, por meio das EqSF, uma vez que a maioria dos municípios são formalmente cobertos pela ESF e, na maior parte deles, essa é a principal porta de entrada para os usuários do SUS (tabela 1). Todavia, há limitações à posição da EqSF como porta de entrada preferencial a outros pontos da rede pública de assistência e à capacidade de controlar o acesso a outros pontos da rede, de forma a assumir o mecanismo gatekeeping como coordenador à atenção especializada, ou seja, tenham profissionais capazes de resolver a maioria dos problemas no âmbito da APS e partilhem o cuidado com serviços de outros níveis de atenção apenas sobre os casos realmente necessários.

Tabela 1. População coberta por Estratégia Saúde da Família (ESF) e quantitativo de Equipes Saúde da Família (EqSF),

Equipes de Saúde Bucal (EqSB) e Núcleo de Apoio à Saúde da Família (Nasf) nos municípios da região de saúde de Vitória da Conquista, Bahia, 2012

\begin{tabular}{|c|c|c|c|c|c|c|c|}
\hline Município & População & $\begin{array}{c}\text { Estimativa } \\
\text { populaccão } \\
\text { coberta }\end{array}$ & № EqSF & $\begin{array}{l}\text { Proporção } \\
\text { cobertura }\end{array}$ & № EqSB & $\begin{array}{c}\text { Razão } \\
\text { EqSB/ESF }\end{array}$ & № Nasf \\
\hline Anagé & 25.516 & 24.150 & 7 & 95 & 2 & 0,3 & 1 \\
\hline Barra do Choc̣a & 34.788 & 34.788 & 12 & 100 & 4 & 0,3 & - \\
\hline Belo Campo & 16.021 & 16.021 & 6 & 100 & 2 & 0,3 & - \\
\hline Bom Jesus da Serra & 10.113 & 10.113 & 3 & 100 & 3 & 1,0 & - \\
\hline Caetanos & 13.639 & 13.639 & 4 & 100 & 3 & 0,8 & - \\
\hline Cândido Sales & 27.918 & 20.700 & 6 & 74 & 3 & 0,5 & - \\
\hline Caraíbas & 10.222 & 10.222 & 4 & 100 & 3 & 0,8 & - \\
\hline Condeúba & 16.898 & 16.898 & 6 & 100 & 5 & 0,8 & - \\
\hline Cordeiros & 8.168 & 8.168 & 4 & 100 & 2 & 0,5 & - \\
\hline Encruzilhada & 23.766 & 13.800 & 4 & 58 & 4 & 1,0 & - \\
\hline Maetinga & 7.038 & 7.038 & 4 & 100 & 2 & 0,5 & 1 \\
\hline Mirante & 10.507 & 10.507 & 4 & 100 & 4 & 1,0 & - \\
\hline Piripá & 12.783 & 12.783 & 6 & 100 & 2 & 0,3 & - \\
\hline Planalto & 24.481 & 20.700 & 6 & 85 & 3 & 0,5 & 1 \\
\hline Poções & 44.701 & 34.500 & 10 & 77 & 8 & 0,8 & 1 \\
\hline Pres. Jânio Quadros & 13.652 & 13.652 & 7 & 100 & 3 & 0,4 & 1 \\
\hline Ribeirão do Largo & 8.602 & 8.602 & 4 & 100 & 2 & 0,5 & - \\
\hline Tremedal & 17.029 & 17.029 & 5 & 100 & 3 & 0,6 & 1 \\
\hline Vitória da Conquista & 306.866 & 127.650 & 37 & 42 & 29 & 0,8 & 4 \\
\hline Região de saúde & 632.708 & 420.960 & 139 & 67 & 87 & 0,6 & 10 \\
\hline
\end{tabular}


O primeiro limite é que a ESF não é a única porta de entrada na APS. Muitos municípios, sobretudo aqueles com menor cobertura, ainda possuem Unidades Básicas de Saúde (UBS) 'tradicionais', complementando o acesso da maior parte da população sem adscrição às USF, ofertando consultas ambulatoriais ou serviço de pronto-atendimento. Trata-se de uma entrada via APS e, também, uma possibilidade minimamente racional de encaminhamento para acesso à atenção especializada, quando necessário, enquanto a ESF não atinge uma cobertura mais alargada. Todavia, e essa é a grande distinção, as UBS tradicionais, grosso modo, concentram grande demanda, gerando, por conseguinte, vinculação precária, o que impede o processo de trabalho clínico de lograr relação de longitudinalidade.

Assim, no contexto das EqSF, constata-se uma 'clínica degradada com médico produtor de demandas':

[...] o município contrata um médico para ir uma vez por semana atender [na USF]. O médico pela própria relação médico-paciente, essa coisa de agradar, não está nem aí. O que o paciente quiser, ele dá; para os parentes, para os amigos, ele sai dando [solicitações de exames]. [...] porque é mais fácil pedir todos os exames e dizer assim: 'Eu estou me cobrindo...'; do que colher uma história que demanda mais tempo pra conversar, explicar que não precisa de ressonância para aquilo ali. [...] ele não quer muito conversar não. É a coisa mesmo do pedir e, também, ele está com pressa, pois tem outro emprego. (GM - Ent.9).

Na rede regional de serviços, encontrou-se, no discurso político e em documentos institucionais, a obrigatoriedade de acesso regulado via APS, mas com inúmeras brechas que descaracterizam e comprometem os fluxos organizacionais. Nesse aspecto, profissionais de hospitais, ambulatórios de especialidades, centrais de marcação de consultas, secretarias municipais de saúde e serviços privados são acessados pelos usuários e geram solicitações e encaminhamentos paralelos para diferentes pontos da rede, sobrepondo-se aos profissionais da APS e debilitando a posição de filtro da ESF. Desse modo, há o 'acesso direto à rede especializada':

Temos aqui [centro de especialidades] demanda espontânea. Não posso dizer que é por ineficiência da atenção básica, mas por questões operacionais, inclusive por falta de recursos humanos, principalmente, de médico e de enfermagem, alguns pacientes não conseguem ter acesso à rede básica e acabam procurando, por isso, a atenção secundária. Então, nós temos aqui outra porta de entrada, pois fazemos uma triagem de alguns casos graves e acabamos atendendo esse paciente. [...] Os profissionais da atenção especializada dão a solicitação para o paciente, pedem que o paciente faça os exames, retorne para ele mesmo, mas não dão o retorno, por exemplo, por escrito para os médicos de atenção básica [...]. (GM - Ent.8).

A intenção de demarcar o acesso direto a serviços especializados na região deve-se à noção que o atributo da coordenação do cuidado não é alcançado por qualquer profissional que possa solicitar ou encaminhar o usuário para outros serviços, mesmo quando a APS é uma porta de entrada obrigatória, mas com característica traçadora de uma ESF forte, ou seja, quando delega aos profissionais da EqSF não apenas o poder de barrar a livre escolha do usuário, mas atribui, essencialmente, a função de gestor terapêutico no fluxo assistencial.

O maior desafio se estabelece após a solicitação da consulta, exame ou procedimento para serviços especializados por causa do estrangulamento na oferta de vagas frente à pressão das demandas, bem como pela capacidade dos profissionais da ESF de acompanhar o percurso do usuário que consegue iniciar o processo terapêutico fora do âmbito da APS. Dessa forma, impõem-se desafios 
aos gestores e profissionais das EqSF para garantir a resolubilidade e continuidade do cuidado dos usuários em tratamento num território regionalizado, com deficiências comunicacionais entre os níveis e insuficiência de serviços de suporte.

Nesse sentido, os mais prejudicados são usuários que procuram a porta mais acessível para expor sua demanda, muitas vezes, em momentos de grande fragilidade, ficando vulneráveis e, portanto, propensos a serem manipulados, revertendo um direito num suposto favor. Essa evidência ficou explícita nas entrevistas e grupos focais que destacam 'clientelismo político sobrepondo à necessidade', 'direito convertido em favor político', 'apadrinhamento e seleção monetária' e 'manipulação política na oferta de vagas’.

Um grupo focal com profissionais sintetiza essa problemática:

Tive um paciente, uma criança com otite de repetição, encaminhei para o otorrino, mas só tinha vaga daqui há 2 anos e meio, pois é uma vaga/mês, mas tem 49 ou 50 pessoas na frente, então acaba que o paciente não vai levar 2 anos e meio para resolver uma otite. Aí vai num profissional particular. E se o usuário não tiver condições de arcar com os custos recorre à política. Vai atrás de vereador, prefeito, através de fulano e sicrano [...], o que é bem comum, porque pouquíssimos têm condições financeiras de sair daqui. (GF-T2).

O trecho destacado ilustra as fissuras no sistema público de saúde e os ataques que minam a ESF e desrespeitam o direito de cidadania. Apesar disso, entende-se que a aparente conveniência de profissionais e usuários não se dá estritamente pela percepção de 'oportunismo', mas parece tratar, também, de uma questão de 'vida ou morte' para muitos usuários ou de alguma condição de resolubilidade às intervenções clínicas para alguns profissionais frente às interdições nos itinerários regulados. Além disso, encontraram-se na região alguns exemplos que despontam no microprocesso de trabalho das equipes e mostram o esforço de gestores e profissionais pela coordenação e ordenamento dos fluxos mesmo em cenários e condições adversas, sintetizados pela 'negociação entre gestores e médicos pela regulação assistencial' e 'envolvimento das EqSF na reavaliação e priorização das necessidades'.

Um gestor municipal resume esse esforço:

[...] foi feita uma reunião, um trabalho de sensibilização, principalmente com médicos, porque médico, às vezes, para se livrar da cara do paciente falava: 'Quer fazer o quê, uma tomografia? Toma lá!'. Então, foi feito um trabalho com médicos pedindo, por favor, avaliem o paciente antes de pedir o exame e, fora isso, quando esse exame chega aqui à secretaria, ainda, faz uma triagem em cima desse exame. A gente enfrenta alguma dificuldade, pois eles [médicos] não colocavam o motivo da solicitação. Então, a gente falou assim: 'vocês estão prejudicando o paciente, porque quando a gente vai triar e não tem lá o motivo da solicitação, esse paciente vai para o fim da fila'. Então, hoje em dia, já colocam o motivo da solicitação, a gente já consegue triar, vê quem é mais urgente, quem deve ser marcado primeiro, quem pode esperar ou não. (GM - Ent.2).

O excerto destacado mostra estratégias para, minimamente, garantir alguma racionalidade e regulação nos fluxos assistenciais, mas não parecem alterar a gênese dos problemas e, portanto, postergam as soluções e transferem responsabilidades para outras instâncias, permanecendo os usuários à mercê das decisões 'benevolentes' dos gestores e de profissionais a cada novo evento.

\section{2) Mecanismos de coordenação do cuidado}

Para uma mínima priorização das demandas, unidades de APS recebem cotas referentes às consultas, exames e procedimentos de acordo com a oferta mensal disponibilizada 
pela CMC de cada município. As equipes, então, têm que, dentre inúmeras necessidades, indicar os usuários 'eleitos', de acordo com descrição clínica ou destaque de 'urgência' atribuído por profissional. Em razão do número de solicitações não atendidas por falta de vagas, algumas equipes reclassificam periodicamente os usuários nas listas de espera, reavaliando a pertinência do pedido (usuário já resolveu por outras vias, usuário não tem mais o problema que justificava o pedido, usuário veio a óbito etc.) para tentar manter certa coerência nos encaminhamentos. Tal seleção abre espaço para inúmeros conflitos éticos, colocando profissionais numa situação de constrangimento e usuários numa clara mitigação da cidadania. Por outro lado, intenta, num cenário de restrição de oferta, minimizar as iniquidades ou, pelo menos, priorizar casos mais urgentes.

O relato do grupo focal com trabalhadores destaca o 'acesso racionado pela oferta e não definido pela necessidade':

[...] a demanda de ultrassonografia é muito grande e o quantitativo de vagas oferecido é muito pouco. Tem que ficar selecionando. $\mathrm{Na}$ unidade que eu trabalho é assim, exames com mais de seis meses, peço para fazer uma reavaliação médica para ver se realmente aquele paciente necessita fazer aquele exame. E conto muito com a ajuda dos ACS [Agentes Comunitários de Saúde], geralmente numa reunião, pegamos os exames, vai passando entre os ACS, eles vão relatando caso a caso, pois, às vezes, a pessoa já marcou. [...], a gente vai fazendo a triagem daqueles exames mais necessários e descartando alguns que não tem tanta necessidade [...]. (GF-T3).

Outra questão que interfere na coordenação entre os níveis assenta-se nas ferramentas para fluxo informacional. Em relação à informatização das USF, além de ser parcial, quando existente, limita-se à marcação de consultas segundo cotas pré-estabelecidas, ou seja, não se trata de fluxo comunicacional entre profissionais para resolução ou acompanhamento de usuários em tratamento. Não há prontuários eletrônicos em nenhum dos estabelecimentos de saúde na região, sendo que as solicitações e referências, muitas vezes, são manuscritas, gerando equívocos nos fluxos, além da ausência de contrarreferências e relatórios de alta hospitalar que permitam aos profissionais das EqSF obter informações técnicas da terapêutica desenvolvida por outros profissionais. Para consecução de informação entre os níveis, o usuário é, na maioria das vezes, o principal interlocutor, quando lhe é permitido falar. Em muitas situações, também, o usuário passa a ficar vinculado ao médico especialista, com retornos frequentes, em vez de criar um vínculo colaborativo entre profissionais da atenção especializada e da ESF, impossibilitando que o médico da USF tenha informações e dê seguimento ao tratamento, além de afunilar a oferta de vagas para novos casos que necessitam de consultas especializadas.

Tal dificuldade no processo de coordenação culmina com a 'filiação do usuário ao especialista', 'comunicação precária entre os níveis' e 'quebra de continuidade no cuidado':

[...] o grande problema do PSF [Programa Saúde da Família] é a contrarreferência, pois não existe. Quando o médico do PSF transfere o paciente para passar por um especialista, fica sem saber o que aconteceu, pois ele não tem o retorno, então, o ideal para que a ESF tivesse uma consolidação boa seria a contrarreferência. [...] Mas quando o paciente chega lá, o cardiologista pega o problema para si e de lá o paciente já sai ciente que não vai mais ao médico do PSF, que o médico que tem que atendê-lo é só aquele que atendeu lá [na especialidade], que trocou a medicação toda. Fica filiado com esse especialista. Aí, a consulta de retorno estrangula. Estrangula o sistema todo, porque o especialista quer a consulta de retorno, sendo que poderia fazer 
um relatório para o médico que encaminhou e remeter para ele o acompanhamento, orientando como [médico de PSF] deve acompanhar, mas isso não existe. (GM - Ent.1).

Contudo, nos pequenos municípios, algumas vezes, o médico da ESF é, também, o prestador de serviços especializados ou também atende nos serviços de pronto-atendimento e hospitalares. Dessa forma, acaba recebendo muitos usuários da própria equipe, além de estar em contato direto com outros especialistas, permitindo algum nível informal de comunicação. Devido aos inúmeros entraves e na busca de alguma coordenação, profissionais lançam mão, muitas vezes, da persuasão e amizade com colegas da rede para tentarem solucionar algumas questões que ficam emperradas na burocracia dos fluxos assistenciais. De maneira geral, tais atitudes partem dos profissionais que estão mais tempo nos serviços, que conhecem as brechas e as pessoas estratégicas nos diferentes pontos da rede, que têm forte vínculo com a comunidade e, portanto, não se omitem diante das negativas burocráticas e preferem tentar resolver por outras vias, ou seja, contraditoriamente, são ações desencadeadas por profissionais mais envolvidos com o processo terapêutico dos usuários.

Nessa direção, alguns profissionais viabilizam o acesso de usuários por meio de 'comunicação condicionada à amizade' e 'empenho pessoal para garantia do acesso':

[...] tenho uma coisinha tão simples que é esse telefone que tem os números de todo mundo. [...] Porque, às vezes, eu resolvo aqui, na hora. Isso as pessoas acham que foi uma coisa do outro mundo, mas é que você pode resolver só com um telefonema. Alguns exames que estão emperrados, algumas coisas você liga direto para a central de marcação, liga para a farmácia. (GF-T4).

Além dos profissionais da ESF, as interferências no fluxo assistencial envolvem profissionais das CMC, das secretarias de saúde e dos centros de especialidades, que, seja por pressão de políticos, profissionais e usuários ou por tentativa simplesmente de interferir em situações clínicas que não podem seguir a 'neutralidade' gerencial, criam novos circuitos de acesso, que, no final, afetam a lógica na distribuição de cotas e retroalimentam o contingenciamento da oferta aos que não gozam dos mesmos privilégios, inclusive por trâmites judiciais. Um problema dessas intervenções paralelas aos fluxos talvez esteja na competência técnica de quem realiza, posto que, em vez de 'boa vontade', há necessidade de diretrizes clínicas e linhas do cuidado bem definidas que garantam segurança na regulação assistencial.

Os registros da observação de campo de visitas à CMC e secretarias municipais de saúde mostram o fluxo contínuo de usuários procurando atendimento em especialidades mesmo sem a solicitação médica (CMC e secretarias municipais como portas de entrada para acessar outros níveis); tentativa (de profissionais e usuários) de antecipação de consultas via 'técnico-marcador' ou coordenador da CMC (independente da lista de espera); coordenadores ou 'técnicos-marcadores' de CMC 'regulando' às prioridades (nem sempre por critérios clínicos); CMC com marcação direta, via guia de solicitação de consultas ou exames, para as especialidades por ordem de chegada do usuário no balcão (sem cotas para USF, nem critério de prioridade para usuários); usuários que se dirigem à CMC com 'bilhetes' de políticos locais para facilitação do fluxo etc. Os casos descritos exemplificam as alternativas legítimas, ou não, que os sujeitos encontram para acessar os serviços de saúde. Há, nessas escolhas, uma composição de favorecimentos que cinde a coordenação por EqSF, mas, paradoxalmente, elas aparecem, também, como mecanismos de coordenação para os casos urgentes represados pela burocrática. 
Assim sendo, constatou-se a intervenção de alguns profissionais para garantir atenção a casos prioritários, ou seja, uma condução profissional orientada mais pela necessidade clínica do usuário e mais vinculada à ética profissional do que às normas e regras que pautam a racionalidade organizacional:

[...] quando o usuário precisa muito daquele exame e tem restrição de vagas, nesse caso, [...] a gente [equipe PSF] joga no sistema, mas também entra em contato com a coordenadora da CMC: 'Coordenadora, esse paciente está assim, ele tem necessidade de fazer esse exame'. Ou a gente encaminha esse paciente direto para o secretário de saúde para avaliar a situação e ver a possibilidade de liberar esses exames que são mais necessários para o paciente. Geralmente, aciono a minha coordenadora, a enfermeira, e digo o problema, às vezes, chego a colocar urgência ou, às vezes, prioridade no pedido [...]. (GF-T3).

A despeito disso, é importante não perder de vista que a oferta de vagas é pré-definida e limitada, portanto, que toda priorização ou privilégio, ainda que legítimo, resulta numa diminuição da cota a ser distribuída aos demais cidadãos (dilema da regulação). Assim, o discurso anterior destaca-se pela coerência em oportunizar o cuidado a quem realmente precisa, mas, paradoxalmente, gera iniquidades àqueles que não foram captados em tempo oportuno (justamente pela ausência de vaga), àqueles que não tiveram 'a sorte' de serem atendidos por um profissional mais pró-ativo ou influente na rede, àqueles que têm suas consultas retardadas por não serem 'prioridade' naquele momento, o que acaba por comprometer o diagnóstico precoce, tornando-se adiante mais uma prioridade, desenvolvendo, assim, um 'círculo vicioso'.

Por fim, uma questão comum que afeta a coordenação reside na longitudinalidade do cuidado pelo mesmo profissional da ESF, sua competência clínica e suporte tecnológico para encaminhar a população adequadamente a outros pontos da rede, em diferentes fases da vida e em distintas situações de enfermidades. Nas EqSF da região, devido à rotatividade de profissionais médicos e contratação de profissionais sem experiência, com idade avançada, sem perfil ou sem formação para trabalhar na APS, há um claro descompasso entre o que poderia ser resolvido nas USF e o que é encaminhado para outros pontos da rede.

\section{Discussão}

A posição da ESF na rede regionalizada como 'centro intercambiador' (MENDES, 2010), lócus de primeiro contato e de procura regular, com profissionais acessíveis para a maioria das necessidades de saúde da população (STARFIELD, 2002), é limitada na região. A baixa cobertura real da ESF, a disponibilidade restrita de médicos, o insuficiente adensamento tecnológico das USF, a escassa oferta de serviços especializados em tempo oportuno e a 'matriz tecnológica' centrada em procedimentos (MERHY, 2002) subjacente ao modelo de atenção em curso na região de saúde limitam a centralidade da APS na rede. $\mathrm{O}$ modelo de atenção mostrou-se, ainda, procedimento-centrado e focado na doença, em detrimento do cuidado centrado na pessoa, conformando uma 'clínica degradada' (CAMPOS, 2003).

Ainda assim, o percurso mais comum do usuário do SUS da região para acessar os serviços de especialidades e apoio diagnóstico e terapêutico é por meio dos estabelecimentos de APS. Mesmo não sendo única porta de entrada ou serviço de busca regular utilizado pelas pessoas para muitas das necessidades incluídas no escopo da APS, a exigência formal de uma 'guia de solicitação' assinada por um médico da ESF para acessar os demais pontos de atenção e a capilaridade das unidades no território tornam-nas os espaços mais acessíveis. 
No caso estudado, os profissionais da ESF, sobretudo médicos, são responsáveis pelas referências para outros pontos de atenção na rede SUS por meio de 'guias de solicitação' e mediados por CMC municipais ou regionais. Por sua vez, as centrais restringem a referência à racionalidade organizacional em detrimento, muitas vezes, da necessidade de saúde dos usuários. Tal evidência atesta que profissionais da APS da região pesquisada agem como gatekeeper do tipo 'regulador burocrático' no sentido da obrigatoriedade de sua referência para que usuários possam acessar outros pontos mais adensados da rede, entretanto, sem lograr mecanismos articulados entre os profissionais dos diferentes níveis, ou seja, sem continuidade e sem coordenação do cuidado (gestão clínica), ficando limitados à função burocrática e constrangidos pela insuficiência de vagas.

Tais características não permitem o desenvolvimento adequado de mecanismo gatekeeping do tipo 'coordenador do cuidado', posto que 'filtrar as demandas e coordená-las' requer condução clínica atenta às necessidades de saúde das pessoas, informações pregressas acerca da histórica clínica dos usuários, responsabilidade terapêutica por um grupo de sujeitos que acessam o serviço regularmente e relação de confiança mútua. Por essa lógica, também, entende-se que muitas EqSF, com todos os problemas elencados, não assumem e não têm condições de assegurar adequadamente $\mathrm{o}$ atributo da coordenação, até porque pouco se distinguem das unidades de saúde tradicionais. Nesse sentido, legitimam a necessidade de outras portas de entrada em detrimento da ESF.

Assim, a questão de coordenação do cuidado e o mecanismo de gatekeeping no Brasil tornam-se desafiadores, posto que, para trabalhar nas EqSF, não há exigência de formação médica específica. Nesse sentido, um médico recém-formado ou especialista em qualquer área pode exercer a função de 'médico da família ou generalista', conformando a ESF num mosaico de profissionais com (ou sem) distintas especialidades (ESCOREL ET AL., 2007), mas frequentemente insuficientes para lidar com a complexidade da APS e, por conseguinte, com grande variabilidade na capacidade resolutiva e de encaminhamento para outros pontos da rede. Ademais, evidências mostram que a oferta e distribuição de médicos de família, o tempo de sua consulta, a oferta de atenção primária (ou serviço de suporte) em horários alternativos e a abrangência dos serviços ofertados são características fundamentais que afetam os resultados da APS (KRINGOS ET AL., 2010).

A organização da ESF na região apresentou questões problemáticas relacionadas, também, à insuficiência de cobertura real e elevado número de pessoas cadastradas por EqSF, que, quando atrelados à baixa disponibilidade de médicos, dificultam o acesso oportuno de usuários aos serviços de APS, a despeito de a cobertura formal da estratégia estar acima de $80 \%$ em 15 dos 19 municípios (tabela 1), indicando que coberturas altas e potencialmente satisfatórias nem sempre se traduzem em melhor utilização da capacidade disponível (CARDOSO; VIEIRA-DA-SILVA, 2007). Vale notar, contudo, que a própria expansão das equipes fica limitada pela baixa oferta de médicos e insuficiência no financiamento, as quais, paradoxalmente, são agravadas com gastos com pagamento de salários de profissionais e pelos limites impostos pela Lei de Responsabilidade Fiscal (NEY; RODRIGUES, 2012). Não por acaso, no Brasil, mesmo em territórios cobertos formalmente com EqSF, o primeiro acesso do usuário ocorre, frequentemente, também via emergência hospitalar e unidades de pronto-atendimento (ALMEIDA; SANTOS; SOUZA, 2015).

\section{Considerações finais}

$\mathrm{Na}$ região estudada, superpõem-se três questões críticas da gestão do trabalho 
médico no SUS que interferem na coordenação do cuidado e no mecanismo gatekeeping: (i) a insuficiência do número de médicos em relação ao total da população, (ii) a distribuição desigual dos médicos no território regionalizado e (iii) a inadequada formação médica para atuação na ESF. Além disso, ressaltamos que a função de gatekeeper em si não garante a coordenação do cuidado, pois a necessidade de saúde do paciente é mais importante do que a racionalidade organizacional.

Na região, os fluxos comunicacionais incipientes entre os diferentes pontos da rede não viabilizam a coordenação do cuidado por profissionais da APS. Sendo assim, a participação das EqSF na regulação do acesso aos serviços especializados é coadjuvante, posto que a priorização é fruto de uma contingência na oferta que, aliada à incipiência de instrumentos para continuidade informacional não contempla a coordenação entre níveis nem a gestão clínica. Tais situações corroboram uma rede fragmentada, uma APS ocupando uma posição marginal e equipes com restrições para coordenar o cuidado.

Como contribuição, entende-se que investigar os limites e possibilidades de coordenação do cuidado em contextos de redes regionalizadas traz importantes indicativos para formulação ou readequação de políticas de saúde, uma vez que os estudos acerca da coordenação do cuidado, de maneira geral, abordam a relação entre profissionais das equipes de APS e profissionais de outros serviços de especialidades e hospitalares dentro do mesmo município.

Ressalta-se, como limites do estudo, que a opção pela pesquisa qualitativa não é suficiente para análise de todos os elementos

\section{Referências}

de uma rede regionalizada para compreensão da coordenação do cuidado, requerendo outras abordagens para ampliar o escopo dos dados e, assim, possibilitar uma triangulação de métodos. Com efeito, a utilização de inquéritos com trabalhadores da saúde e usuários poderia trazer informações relevantes sobre acesso e utilização dos serviços em diferentes pontos da rede, encaminhamentos e tempos de espera, bem como sobre condições estruturais e insumos disponíveis em estabelecimentos de saúde no conjunto dos municípios da região. Ademais, a constelação dos sujeitos participantes pode ser ampliada para outros segmentos da rede, conforme o foco desejado, e, igualmente, captar perspectivas que respondam a aspectos complementares à coordenação do cuidado. Pode-se, também, ampliar o estudo para diferentes regiões de saúde e realizar uma análise comparada entre elas, aumentando a validade externa.

Por fim, os achados podem ser úteis para se avaliar a coordenação do cuidado em outras regiões de saúde, pois o estudo abarcou as estratégias entre um município de médio porte e municípios de pequeno porte (com populações rurais e rarefeitas), evidenciando as peculiaridades para atração e fixação de profissionais nessas localidades e os desafios para provimento de serviços de saúde. Nesse sentido, indica a necessidade de políticas específicas para os territórios que admitem outros arranjos no número e na composição das EqSF, valorizando e ampliando, quando possível e pertinente, a atuação do enfermeiro, de equipes do Núcleo de Apoio à Saúde da Família (ou outras composições), dos técnicos e dos agentes comunitários de saúde. paralelos entre Brasil e Espanha. Saúde Debate, Rio de Janeiro, v. 37, n. 98, p. 400-415, 2013. 
ALMEIDA, P. F.; SANTOS, A. M.; SOUZA, M. K. B. Atenção Primária à Saúde na coordenação do cuidado em Regiões de Saúde. Salvador: Edufba, 2015.

BAHIA. Secretaria da Saúde. Resolução CIB 275/2012. Aprova as regiões de saúde do Estado da Bahia e a instituição das Comissões Intergestores Regionais. Diário Oficial [do] Estado da Bahia, Salvador: SESAB, 2012. Disponível em: <http://www2.saude.ba.gov.br/ cib/arquivos/Resolu\%C3\%A7\%C3\%B5es\%20da\%20 CIB/2012/Resolu\%C3\%A7\%C3\%B5es\%20CIB\%20 de\%20151\%20a\%20300.pdf>. Acesso em: 14 out. 2015.

BRASIL. Ministério da Saúde. Decreto 7.508, de 28 de junho de 2011. Regulamenta a lei $\mathrm{n}^{\circ}$. 8.080, de 19 de setembro de 1990, para dispor sobre a organização do SUS, o planejamento da saúde, a assistência à saúde e a articulação interfederativa, e dá outras providências. Diário Oficial [da] União, Brasília, DF, 2011. Disponível em: <http://www.planalto.gov.br/ccivil_03/_ato20112014/2011/decreto/D7508.htm>. Acesso em: 14 out. 2015

CAMPOS, G. W. S. Saúde Paidéia. São Paulo: Hucitec, 2003.

CARDOSO, M. O.; VIEIRA-DA-SILVA, L. M. Avaliação da cobertura da atenção básica à saúde em Salvador, Bahia, Brasil (2000 a 2007). Cad. Saúde Pública, Rio de Janeiro, v. 28, n. 7, p. 1273-1284, 2012.

CONILL, E. M.; FAUSTO, M. C. R.; GIOVANELLA, L. Contribuições da análise comparada para um marco abrangente na avaliação de sistemas orientados pela atenção primária na América Latina. Rev Bras Saude Matern. Infant., Recife, v. 10, supl. 10, p. 15-27, 2010.

ESCOREL, S. et al. O Programa de Saúde da Família e a construção de um novo modelo para a Atenção Básica no Brasil. Rev Panam Salud Publica, Washington, v. 21, n. 2, p. 164-176, 2007.

GARRIDO, M. V.; ZENTNER, A.; BUSSE, R. The effects of gatekeeping: a systematic review of the literature. Scandinavian Journal of Primary Health Care, v. 29, n. 1, p. 28-38, 2011. Disponível em: <http://www.tandfonline. com/doi/full/10.3109/02813432.2010.537015>. Acesso em: 27 nov. 2015.
GÉRVAS, J. et al. Algunas causas (y soluciones) de la pérdida de prestigio de la medicina general/de familia. Contra el descrédito del héroe. Aten Primaria, [Internet], v. 39, n. 11, p. 615-18, 2007. Disponível em: <http:// equipocesca.org/new/wp-content/uploads/2009/03/ descredito-del-heroe-2007.pdf >. Acesso em: 27 nov. 2015.

GÉRVAS, J.; PEREZ FERNANDEZ, M. El fundamento científico de La función de filtro del médico general. Rev Bras Epidemiol., São Paulo, v. 8, n. 2, p. 205-18, 2005.

GÉRVAS, J.; RICO, A. La coordinación en el sistema sanitario y su mejora a través de las reformas europeas de la Atención Primaria. SEMERGEN, Madrid, v. 31, n. 9, p. 418-423, 2005.

GIOVANELLA, L. A atenção primária à saúde nos países da União Européia: configurações e reformas organizacionais na década de 1990. Cad. Saúde Pública, Rio de Janeiro, v. 22, n. 5, p. 951-963, 2006.

Redes integradas, programas de gestão clínica e generalista coordenador: análise das reformas recentes do setor ambulatorial na Alemanha. Ciênc. Saúde Coletiva, Rio de Janeiro, v. 16, supl. 1, p. 1081-1096, 2011.

GREß, S. et al. Co-ordination and management of chronic conditions in Europe: the role of primary care position paper of the European Forum for Primary Care. Quality in Primary Care, v. 17, n. 1, p. 75-86, 2009.

HAGGERTY, J. L. et al. Continuity of care: a multidisciplinary review. BMJ, Bethesda, v. 327, p. 12191221,2003

HERNÁNDEZ, M. B. A. La continuidad asistencial entre niveles precibida por usuários del sistema de salud en Cataluña. Revista Española de Salud Publica, Madrid, v. 84, n. 4, p. 371-87, 2010.

HOFMARCHER, M. M.; OXLEY, H.; RUSTICELLI, E. Improved Health System Performance Through Better Care Coordination. OECD Health Working Papers, Paris, n. $30,2007$.

KRINGOS, D. S. et al. The breadth of primary care: a systematic literature review of its core dimensions. 
BMC Health Services Research, v. 10, n. 65, p. 1-13, 2010. Disponível em: <http://www.biomedcentral.com/14726963/10/65>. Acesso em: 27 nov. 2015.

MENDES, E. V. As redes de atenção à saúde. Ciênc Saúde Coletiva, Rio de Janeiro, v. 15, n. 5, p. 2297-2305, 2010.

MERHY, E. E. Saúde a cartografia do trabalho vivo. São Paulo: Hucitec, 2002.

MINAYO, M. C. S. O desafio do conhecimento: pesquisa qualitativa em saúde. Hucitec: São Paulo, 2004.

MUR-VEEMAN, I.; VAN RAAK, A.; PAULUS, A. Comparing integrated policy in Europe: Does policy matter? Health Policy, v. 85, n.2, p. 172-183, 2008.

Disponível em: <http://www.sciencedirect.com/science/ article/pii/S0168851007001728>. Acesso em: 27 nov. 2015 .

NEY, M. S.; RODRIGUES, P. H. A. Fatores críticos para fixação do médico na Estratégia Saúde da Família. Physis, Rio de Janeiro, v. 22, n. 4, p. 1293-311, 2012.

SALTMAN, R. B.; RICO, A.; BOERMA, W. G. W. Atenção Primária conduzindo as redes de atenção à saúde: reforma organizacional na atenção primária europeia. Berkshire:
Open University Press; 2006.

SHOEN, C. et al. A survey of primary care doctors in ten countries shows progress in use of health information technology, less in other areas. Health Affairs, v. 31, n. 12, p. 2805-2816, 2012. Disponível em: <http://content. healthaffairs.org/content/31/12/2805.long $>$. Acesso em: 27 nov. 2015

STARFIELD, B. Atenção primária: equilíbrio entre necessidades de saúde, serviços e tecnologia. Brasília, DF: Unesco: Ministério da Saúde, 2002.

STARFIELD, B.; SHI, L.; MACINKO, J. Contribution of Primary Care to health systems and health. The Milbank Quarterly, Bethesda, v. 83, n. 3, p. 457-502, 2005.

VÁZQUEZ, M. L. et al. Integrated health care network in Latin America: toward a conceptual framework for analysis. Rev Panam Salud Pública, Washington, DC, v. 26, n. 4, p. 360-367, 2009.

Recebido para publicação em junho de 2015

Versão final em outubro de 2015

Conflito de interesses: inexistente

Suporte financeiro: não houve 